\title{
Accelerated Circulant and Skew Circulant Splitting Methods for Hermitian Positive Definite Toeplitz Systems
}

\author{
N. Akhondi and F. Toutounian \\ School of Mathematical Sciences, Ferdowsi University of Mashhad, P.O. Box 1159-91775, \\ Mashhad 9177948953, Iran \\ Correspondence should be addressed to N. Akhondi, nasserakhoundi@gmail.com
}

Received 5 August 2011; Revised 26 October 2011; Accepted 26 October 2011

Academic Editor: Ivan Ganchev Ivanov

Copyright (C 2012 N. Akhondi and F. Toutounian. This is an open access article distributed under the Creative Commons Attribution License, which permits unrestricted use, distribution, and reproduction in any medium, provided the original work is properly cited.

\begin{abstract}
We study the CSCS method for large Hermitian positive definite Toeplitz linear systems, which first appears in $\mathrm{Ng}^{\prime}$ s paper published in $(\mathrm{Ng}, 2003)$, and CSCS stands for circulant and skew circulant splitting of the coefficient matrix $A$. In this paper, we present a new iteration method for the numerical solution of Hermitian positive definite Toeplitz systems of linear equations. The method is a two-parameter generation of the CSCS method such that when the two parameters involved are equal, it coincides with the CSCS method. We discuss the convergence property and optimal parameters of this method. Finally, we extend our method to BTTB matrices. Numerical experiments are presented to show the effectiveness of our new method.
\end{abstract}

\section{Introduction}

We consider iterative solution of the large system of linear equations

$$
A x=b,
$$

where $A \in \mathbb{C}^{n \times n}$ is Hermitian positive definite Toeplitz matrix and $b, x \in \mathbb{C}^{n}$. An $n$-by$n$ matrix $A=\left(a_{i, j}\right)_{i, j=1}^{n}$ is said to be Toeplitz if $a_{i, j}=a_{i-j}$; that is, $A$ is constant along its diagonals. Toeplitz systems arise in a variety of applications, especially in signal processing and control theory. Many direct methods are proposed for solving Toeplitz linear systems. A straightforward application of Gaussian elimination will lead to an algorithm with $O\left(n^{3}\right)$ complexity. There are a number of fast Toeplitz solvers that decrease the complexity to $O\left(n^{2}\right)$ operations, see for instance [1-3]. Around 1980, superfast direct Toeplitz solvers of 
complexity $O\left(n \log ^{2} n\right)$, such as the one by Ammar and Gragg [4], were also developed. Recent research on using the preconditioned conjugate gradient method as an iterative method for solving Toeplitz systems has brought much attention. One of the main important results of this methodology is that the PCG method has a computational complexity proportional to $O(n \log n)$ for a large class of problem [5] and is therefore competitive with any direct method.

In [6], an iterative method based on circulant and skew circulant splitting (CSCS) of the Toeplitz matrix was given. The authors have driven an upper bound of the contraction factor of the CSCS iteration which is dependent solely on the spectra of the circulant and the skew circulant matrices involved.

In [7] the authors studied the HSS iteration method for large sparse non-Hermitian positive definite Toeplitz linear systems, which first appears in [8]. They used the HSS iteration method based on a special case of the HSS splitting, where the symmetric part $H=(1 / 2)\left(A+A^{T}\right)$ is a centrosymmetric matrix and skew-symmetric part $S=(1 / 2)\left(A-A^{T}\right)$ is a skew-centrosymmetric matrix for a given Toeplitz matrix and discussed the computational complexity of the HSS and IHSS methods.

In this paper we present an efficient iterative method for the numerical solution of Hermitian positive definite Toeplitz systems of linear equations. The method is a twoparameter generation of the CSCS method such that when the two parameters involved are equal, it coincides with the CSCS method. We discuss the convergence property and optimal parameters of this method. Then we extend our method to block-Toeplitz-Toeplitz-block (BTTB) matrices.

For convenience, some of the terminology used in this paper will be given. The symbol $\mathbb{C}^{n \times n}$ will denote the set of all $n \times n$ complex matrices. Let $A, B \in \mathbb{C}^{n \times n}$. We use the notation $A>0 \quad(A \geq 0)$ if $A$ is Hermitian positive (semi-)definite. If $A$ and $B$ are both Hermitian, we write $A>B \quad(A \geq B)$ if and only if $A-B>0 \quad(A-B \geq 0)$. For a Hermitian positive definite matrix $A$, we define the $\|\cdot\|_{A}$ norm of a vector $z \in \mathbb{C}^{n}$ as $\|z\|_{A}=\sqrt{z^{*} A z}$. Then the induced $\|\cdot\|_{A}$ norm of a matrix $B \in \mathbb{C}^{n \times n}$ is defined as $\|B\|_{A}=\left\|A^{1 / 2} B A^{-1 / 2}\right\|_{A}$.

The organization of this paper is as follows. In Section 2, we present accelerated circulant and skew circulant splitting (ACSCS) method for Toeplitz systems. In Section 3, we study the convergence properties and analyze the convergence rate of ACSCS iteration and derive optimal parameters. The convergence results of ACSCS method for BTTB matrices are given in Section 4. Numerical experiments are presented in Section 5 to show the effectiveness of our new method. Finally some conclusions are given in Section 6.

\section{Accelerated Circulant and Skew Circulant Splitting Method}

Let us begin by supposing that the entries $a_{i, j}=a_{i-j}$ of $n$-by- $n$ Toeplitz matrix $A_{n}(=A)$ are the Fourier coefficients of the real generating function

$$
f(\theta)=\sum_{-\infty}^{\infty} a_{k} e^{-i k \theta}
$$

defined on $[-\pi, \pi]$. Since $f$ is a real-valued function, $a_{-k}=\bar{a}_{k}$ for all integers and $A_{n}$ is a Hermitian matrix. For Hermitian Toeplitz matrix $A_{n}$ we note that it can always be split as

$$
A_{n}=C_{n}+S_{n}
$$


where

$$
\begin{gathered}
C_{n}=\frac{1}{2}\left(\begin{array}{ccccc}
a_{0} & a_{-1}+a_{n-1} & & & a_{-(n-1)}+a_{1} \\
a_{1}+a_{-(n-1)} & a_{0} & & & \\
& & \ddots & \ddots & \\
& & \ddots & \ddots & a_{-1}+a_{n-1} \\
a_{(n-1)}+a_{-1} & & & & a_{0}
\end{array}\right), \\
S_{n}=\frac{1}{2}\left(\begin{array}{ccccc}
a_{0} & a_{-1}-a_{n-1} & & a_{-(n-1)}-a_{1} \\
a_{1}-a_{-(n-1)} & a_{0} & & & \\
& & \ddots & \ddots & \\
& & \ddots & \ddots & a_{-1}-a_{n-1} \\
a_{(n-1)}-a_{-1} & & & & a_{0}
\end{array}\right) .
\end{gathered}
$$

Clearly $C_{n}$ is Hermitian circulant matrix and $S_{n}$ is Hermitian skew circulant matrix. The positive definiteness of $C_{n}$ and $S_{n}$ is given in the following theorem. Its proof is similar to that of Theorem 2 in [9].

Theorem 2.1. Let $f$ be a real-valued function in the Wiener class $\left(\sum_{-\infty}^{\infty}\left|a_{k}\right|<\infty\right)$ and satisfies the condition

$$
f(\theta)=\sum_{-\infty}^{\infty} a_{k} e^{-i k \theta} \geq \delta>0 \quad \forall \theta
$$

Then the circulant matrix $C_{n}$ and the skew circulant matrix $S_{n}$, defining by the splitting $A_{n}=C_{n}+S_{n}$, are uniformly positive and bounded for sufficiently large $n$.

The subscript $n$ of matrices is omitted hereafter whenever there is no confusion.

Based on the splitting (2.2), $\mathrm{Ng}$ [6] presented the CSCS iteration method: given an initial guess $x^{(0)}$, for $k=0,1,2, \ldots$, until $x^{(k)}$ converges, compute

$$
\begin{gathered}
(\alpha I+C) x^{(k+1 / 2)}=(\alpha I-S) x^{(k)}+b, \\
(\alpha I+S) x^{(k+1)}=(\alpha I-C) x^{(k+1 / 2)}+b,
\end{gathered}
$$

where $\alpha$ is a given positive constant. He has also proved that if the circulant and the skew circulant splitting matrices are positive definite, then the CSCS method converges to the unique solution of the system of linear equations. Moreover, he derived an upper bound of the contraction of the CSCS iteration which is dependent solely on the spectra of the circulant and the skew circulant matrices $C$ and $S$, respectively.

In this paper, based on the CSCS splitting, we present a different approach to solve (1.1) with the Hermitian positive definite coefficient matrix, called the Accelerated Circulant 
and Skew Circulant Splitting method, shortened to the ACSCS iteration. Let us describe it as follows.

The ACSCS iteration method: given an initial guess $x^{(0)}$, for $k=0,1,2, \ldots$, until $x^{(k)}$ converges, compute

$$
\begin{gathered}
(\alpha I+C) x^{(k+1 / 2)}=(\alpha I-S) x^{(k)}+b \\
(\beta I+S) x^{(k+1)}=(\beta I-S) x^{(k+1 / 2)}+b
\end{gathered}
$$

where $\alpha$ is a given nonnegative constant and $\beta$ is given positive constant.

The ACSCS iteration alternates between the circulant matrix $C$ and the skew circulant matrix $S$. Theoretical analysis shows that if the coefficient matrix $A$ is Hermitian positive definite the ACSCS iteration (2.6) can converge to the unique solution of linear system (1.1) with any given nonnegative $\alpha$, if $\beta$ is restricted in an appropriate region. And the upper bound of contraction factor of the ACSCS iteration is dependent on the choice of $\alpha, \beta$, the spectra of the circulant matrix $C$, and the skew circulant matrix $S$. The two steps at each ACSCS iterate require exact solutions with the $n \times n$ matrices $\alpha I+C$ and $\beta I+S$. Since circulant matrices can be diagonalized by the discrete Fourier matrix $F$ and skew circulant matrices can be diagonalized by the diagonal times discrete Fourier $\widehat{F}[10]$, that is,

$$
C=F^{*} \Lambda_{1} F, \quad S=\widehat{F}^{*} \Lambda_{2} \widehat{F},
$$

where $\Lambda_{1}$ and $\Lambda_{2}$ are diagonal matrices holding the eigenvalues of $C$ and $S$, respectively, the exact solutions with circulant matrices and skew circulant matrices can be obtained by using fast Fourier transforms (FFTs). In particular, the number of operations required for each step of the ACSCS iteration method is $O(n \log n)$.

Noting that the roles of the matrices $C$ and $S$ in (2.6) can be reverse, we can first solve the system of linear equation with the $\beta I+S$ and then solve the system of linear equation with coefficient matrix $\alpha I+C$.

\section{Convergence Analysis of the ACSCS Iteration}

In this section we study the convergence rate of the ACSCS iteration and we suppose that the entries $a_{i, j}=a_{i-j}$ of $A$ are the Fourier coefficient of the real generating function $f$ that satisfies the conditions of Theorem 2.1. So, for sufficiently large $n$, the matrices $A, C$, and $S$ will be Hermitian positive definite. Let us denote the eigenvalues of $C$ and $S$ by $\lambda_{i}, \mu_{i}, i=$ $1, \ldots, n$, and the minimum and maximum eigenvalues of $C$ and $S$ by $\lambda_{\min }, \lambda_{\max }$ and $\mu_{\min }, \mu_{\max }$, respectively. Therefore, from Theorem 2.1, for sufficiently large $n$ we have $\lambda_{\min }>0$ and $\mu_{\min }>$ 0.

We first note that the ACSCS iteration method can be generalized to the two-step splitting iteration framework, and the following lemma describes a general convergence criterion for a two-step splitting iteration.

Lemma 3.1. Let $A \in \mathbb{C}^{n \times n}, A=M_{i}-N_{i}(i=1,2)$ be two splitting of the matrix $A$, and $x^{(0)} \in \mathbb{C}^{n}$ be a given initial vector. If $x^{(k)}$ is a two-step iteration sequence defined by

$$
\begin{gathered}
M_{1} x^{(k+1 / 2)}=N_{1} x^{(k)}+b, \\
M_{2} x^{(k+1)}=N_{2} x^{(k+1 / 2)}+b,
\end{gathered}
$$


$k=0,1,2, \ldots$, then

$$
x^{k+1}=M_{2}^{-1} N_{2} M_{1}^{-1} N_{1} x^{k}+M_{2}^{-1}\left(I+N_{2} M_{1}^{-1}\right) b, \quad k=0,1,2, \ldots
$$

Moreover, if the spectral radius $\rho\left(M_{2}^{-1} N_{2} M_{1}^{-1} N_{1}\right)<1$, then the iterative sequence $x^{(k)}$ converges to the unique solution $x^{*} \in \mathbb{C}^{n}$ of the system of linear equations (1.1) for all initial vectors $x^{(0)} \in \mathbb{C}^{n}$.

Applying this lemma to the ACSCS iteration, we obtain the following convergence property.

Theorem 3.2. Let $A \in \mathbb{C}^{(n \times n)}$ be a Hermitian positive definite Toeplitz matrix, and let $C, S$ be its Hermitian positive circulant and skew circulant parts, $\alpha$ be a nonnegative constant, and $\beta$ be a positive constant. Then the iteration matrix $M(\alpha, \beta)$ of the ACSCS method is

$$
M(\alpha, \beta)=(\beta I+S)^{-1}(\beta I-C)(\alpha I+C)^{-1}(\alpha I-S),
$$

and its spectral radius $\rho(M(\alpha, \beta))$ is bounded by

$$
\delta(\alpha, \beta)=\max _{i=1}^{n}\left|\frac{\beta-\lambda_{i}}{\alpha+\lambda_{i}}\right| \max _{i=1}^{n}\left|\frac{\alpha-\mu_{i}}{\beta+\mu_{i}}\right|,
$$

where $\lambda_{i}, \mu_{i}, i=1, \ldots, n$ are eigenvalues of $C, S$, respectively. And for any given parameter $\alpha$ if

$$
\alpha-2 \mu_{\min }<\beta<\alpha+2 \lambda_{\min }
$$

then $\delta(\alpha, \beta)<1$, that is, the ACSCS iteration converges, where $\lambda_{\min }, \mu_{\min }$ are the minimum eigenvalue of $C$ and $S$, respectively.

Proof. Setting

$$
M_{1}=\alpha I+C, \quad N_{1}=\alpha I-S, \quad M_{2}=\beta I+S, \quad N_{2}=\beta I-C
$$

in Lemma 3.1. Since $\alpha I+C$ and $\beta I+S$ are nonsingular for any nonnegative constant $\alpha$ and positive $\beta$, we get (3.3).

By similarity transformation, we have

$$
\begin{aligned}
\rho(M(\alpha, \beta)) & =\rho\left((\beta I+S)^{-1}(\beta I-C)(\alpha I+C)^{-1}(\alpha I-S)\right) \\
& =\rho\left((\beta I-C)(\alpha I+C)^{-1}(\alpha I-S)(\beta I+S)^{-1}\right) \\
& \leq\left\|(\beta I-C)(\alpha I+C)^{-1}(\alpha I-S)(\beta I+S)^{-1}\right\|_{2} \\
& \leq\left\|(\beta I-C)(\alpha I+C)^{-1}\right\|_{2}\left\|(\alpha I-S)(\beta I+S)^{-1}\right\|_{2} \\
& =\max _{i=1}^{n}\left|\frac{\beta-\lambda_{i}}{\alpha+\lambda_{i}}\right| \max _{i=1}^{n}\left|\frac{\alpha-\mu_{i}}{\beta+\mu_{i}}\right| .
\end{aligned}
$$

Then the bound for $\rho(M(\alpha, \beta))$ is given by (3.4). 
Since $\alpha \geq 0, \beta>0$, and $\beta$ satisfies the relation (3.5), the following equalities hold:

$$
\begin{aligned}
& \max _{i=1}^{n}\left|\frac{\beta-\lambda_{i}}{\alpha+\lambda_{i}}\right|=\max \left(\left|\frac{\beta-\lambda_{\max }}{\alpha+\lambda_{\max }}\right|,\left|\frac{\beta-\lambda_{\min }}{\alpha+\lambda_{\min }}\right|\right)<1, \\
& \max _{i=1}^{n}\left|\frac{\alpha-\mu_{i}}{\beta+\mu_{i}}\right|=\max \left(\left|\frac{\alpha-\mu_{\max }}{\beta+\mu_{\max }}\right|,\left|\frac{\alpha-\mu_{\min }}{\beta+\mu_{\min }}\right|\right)<1,
\end{aligned}
$$

so $\delta(\alpha, \beta)<1$.

Theorem 3.2 mainly discusses the available $\beta$ for a convergent ACSCS iteration for any given nonnegative $\alpha$. It also shows that the choice of $\beta$ is dependent on the minimum eigenvalue of the circulant matrix $C$ and the skew circulant matrix $S$ and the choice of $\alpha$. Notice that

$$
\left(\alpha+2 \lambda_{\min }\right)-\left(\alpha-2 \mu_{\min }\right)=2\left(\lambda_{\min }+\mu_{\min }\right)>0,
$$

then we remark that for any $\alpha$ the available $\beta$ exists. And if $\lambda_{\min }$ and $\mu_{\min }$ are large, then the restriction put on $\beta$ is loose. The bound on $\delta(\alpha, \beta)$ of the convergence rate depends on the spectrum of $C$ and $S$ and the choice of $\alpha$ and $\beta$. Moreover, $\delta(\alpha, \beta)$ is also an upper bound of the contraction of the ACSCS iteration.

Moreover, from the proof of Theorem 3.2 we can simplify the bound $\delta(\alpha, \beta)$ as

$$
\delta(\alpha, \beta)=\max \left(\left|\frac{\beta-\lambda_{\max }}{\alpha+\lambda_{\max }}\right|,\left|\frac{\beta-\lambda_{\min }}{\alpha+\lambda_{\min }}\right|\right) \times \max \left(\left|\frac{\alpha-\mu_{\max }}{\beta+\mu_{\max }}\right|,\left|\frac{\alpha-\mu_{\min }}{\beta+\mu_{\min }}\right|\right) .
$$

In the following lemma, we list some useful relations related to the minimum and maximum eigenvalues of matrices $C$ and $S$, which are essential for us to obtain the optimal parameters $\alpha$ and $\beta$ and to describe their properties.

Lemma 3.3. Let $S_{\lambda}=\lambda_{\min }+\lambda_{\max }, S_{\mu}=\mu_{\min }+\mu_{\max }$ and $P_{\lambda}=\lambda_{\min } \lambda_{\max }, P_{\mu}=\mu_{\min } \mu_{\max }$, then the following relations hold:

$$
\begin{aligned}
\mu_{\max }\left(S_{\mu}+S_{\curlywedge}\right)-\left(P_{\mu}-P_{\curlywedge}\right) & =\left(\mu_{\max }+\lambda_{\min }\right)\left(\mu_{\max }+\lambda_{\max }\right), \\
\mu_{\min }\left(S_{\mu}+S_{\curlywedge}\right)-\left(P_{\mu}-P_{\curlywedge}\right)= & \left(\mu_{\min }+\lambda_{\min }\right)\left(\mu_{\min }+\lambda_{\max }\right), \\
\lambda_{\max }\left(S_{\curlywedge}+S_{\mu}\right)-\left(P_{\curlywedge}-P_{\mu}\right) & =\left(\lambda_{\max }+\mu_{\min }\right)\left(\lambda_{\max }+\mu_{\max }\right), \\
\lambda_{\min }\left(S_{\lambda}+S_{\mu}\right)-\left(P_{\lambda}-P_{\mu}\right)= & \left(\lambda_{\min }+\mu_{\min }\right)\left(\lambda_{\min }+\mu_{\max }\right), \\
\left(P_{\mu}-P_{\lambda}\right)^{2}+\left(S_{\curlywedge}+S_{\mu}\right)\left(S_{\mu} P_{\lambda}+S_{\curlywedge} P_{\mu}\right)= & \left(\lambda_{\max }+\mu_{\min }\right)\left(\mu_{\max }+\lambda_{\min }\right) \\
& \times\left(\lambda_{\max }+\mu_{\max }\right)\left(\lambda_{\min }+\mu_{\min }\right) .
\end{aligned}
$$

Proof. The equalities follow from straightforward computation. 
Theorem 3.4. Let $A, C, S$ be the matrices defined in Theorem 3.2 and $S_{\lambda}, S_{\mu}, P_{\lambda}, P_{\mu}$ be defined as Lemma 3.3. Then the optimal $\alpha^{*}, \beta^{*}$ should be

$$
\begin{aligned}
& \alpha^{*}=\frac{\left(P_{\mu}-P_{\lambda}\right)+\sqrt{\left(P_{\mu}-P_{\lambda}\right)^{2}+\left(S_{\mu}+S_{\lambda}\right)\left(S_{\mu} P_{\lambda}+S_{\lambda} P_{\mu}\right)}}{S_{\mu}+S_{\lambda}}, \\
& \beta^{*}=\frac{\left(P_{\lambda}-P_{\mu}\right)+\sqrt{\left(P_{\mu}-P_{\lambda}\right)^{2}+\left(S_{\mu}+S_{\lambda}\right)\left(S_{\mu} P_{\lambda}+S_{\lambda} P_{\mu}\right)}}{S_{\mu}+S_{\lambda}},
\end{aligned}
$$

and they satisfy the relations

$$
\begin{gathered}
\mu_{\min }<\alpha^{*}<\mu_{\max } \\
\lambda_{\min }<\beta^{*}<\lambda_{\max } \\
\alpha^{*}-2 \mu_{\min }<\beta^{*}<\alpha^{*}+2 \lambda_{\min } .
\end{gathered}
$$

And the optimal bound is

$$
\delta^{*}\left(\alpha^{*}, \beta^{*}\right)=\frac{\sqrt{\left(\lambda_{\max }+\mu_{\min }\right)\left(\mu_{\max }+\lambda_{\min }\right) /\left(\lambda_{\max }+\mu_{\max }\right)\left(\lambda_{\min }+\mu_{\min }\right)}-1}{\sqrt{\left(\lambda_{\max }+\mu_{\min }\right)\left(\mu_{\max }+\lambda_{\min }\right) /\left(\lambda_{\max }+\mu_{\max }\right)\left(\lambda_{\min }+\mu_{\min }\right)}+1}
$$

Proof. From Theorem 3.2 and (3.8) there exist a $\beta^{*} \in\left[\lambda_{\min }, \lambda_{\max }\right]$ and $\alpha^{*} \in\left[\mu_{\min }, \mu_{\max }\right]$ such that

$$
\begin{gathered}
\max _{i=1}^{n}\left|\frac{\beta-\lambda_{i}}{\alpha+\lambda_{i}}\right|= \begin{cases}\frac{\lambda_{\max }-\beta}{\lambda_{\max }+\alpha}, & \beta \leq \beta^{*}, \\
\frac{\beta-\lambda_{\min }}{\alpha+\lambda_{\min }}, & \beta \geq \beta^{*},\end{cases} \\
\max _{i=1}^{n}\left|\frac{\alpha-\mu_{i}}{\beta+\mu_{i}}\right|= \begin{cases}\frac{\mu_{\max }-\alpha}{\mu_{\max }+\beta}, & \alpha \leq \alpha^{*}, \\
\frac{\alpha-\mu_{\min }}{\beta+\mu_{\min }}, & \alpha \geq \alpha^{*},\end{cases}
\end{gathered}
$$

respectively. In order to minimize the bound in (3.10), the following equalities must hold:

$$
\begin{aligned}
& \frac{\beta-\lambda_{\min }}{\alpha+\lambda_{\min }}=\frac{\lambda_{\max }-\beta}{\lambda_{\max }+\alpha}, \\
& \frac{\alpha-\mu_{\min }}{\beta+\mu_{\min }}=\frac{\mu_{\max }-\alpha}{\mu_{\max }+\beta} .
\end{aligned}
$$


By using $S_{\lambda}=\lambda_{\max }+\lambda_{\min }, P_{\lambda}=\lambda_{\max } \lambda_{\min }, S_{\mu}=\mu_{\max }+\mu_{\min }, P_{\mu}=\mu_{\max } \mu_{\min }$, the above equalities can be rewritten as

$$
\begin{aligned}
& 2\left(\alpha \beta-P_{\lambda}\right)=(\alpha-\beta) S_{\lambda}, \\
& 2\left(\alpha \beta-P_{\mu}\right)=(\beta-\alpha) S_{\mu} .
\end{aligned}
$$

These relations imply that

$$
\alpha-\beta=\frac{2\left(P_{\mu}-P_{\lambda}\right)}{S_{\curlywedge}+S_{\mu}}, \quad \alpha \beta=\frac{S_{\mu} P_{\lambda}+S_{\lambda} P_{\mu}}{S_{\lambda}+S_{\mu}}
$$

By putting $\beta^{\prime}=-\beta$, the parameters $\alpha$ and $\beta^{\prime}$ will be the roots of the quadratic polynomial

$$
x^{2}-\frac{2\left(P_{\mu}-P_{\lambda}\right)}{S_{\mu}+S_{\lambda}} x-\frac{S_{\mu} P_{\lambda}+S_{\lambda} P_{\mu}}{S_{\mu}+S_{\curlywedge}}=0 .
$$

Solving this equation we get the parameters $\alpha^{*}$ and $\beta^{*}$ given by (3.16) and (3.17), respectively. These parameters $\alpha^{*}$ and $\beta^{*}$ can be considered as optimal parameters if they satisfy the relations (3.18)-(3.20).

From (3.12), (3.15) and (3.11), (3.15), we have

$$
\begin{aligned}
& \mu_{\min }\left(S_{\lambda}+S_{\mu}\right)-\left(P_{\mu}-P_{\lambda}\right) \leq \sqrt{\left(P_{\mu}-P_{\lambda}\right)^{2}+\left(S_{\lambda}+S_{\mu}\right)\left(S_{\mu} P_{\lambda}+S_{\lambda} P_{\mu}\right)} \\
& \mu_{\max }\left(S_{\lambda}+S_{\mu}\right)-\left(P_{\mu}-P_{\lambda}\right) \geq \sqrt{\left(P_{\mu}-P_{\lambda}\right)^{2}+\left(S_{\lambda}+S_{\mu}\right)\left(S_{\mu} P_{\lambda}+S_{\lambda} P_{\mu}\right)}
\end{aligned}
$$

respectively. From these inequalities, by the definition of $\alpha^{*}$ and simple computation, we get $\mu_{\min } \leq \alpha^{*} \leq \mu_{\max }$. By similarity computation, we can also show that $\lambda_{\min } \leq \beta^{*} \leq \lambda_{\max }$. So, the parameters $\alpha^{*}$ and $\beta^{*}$ satisfy the relations (3.18) and (3.19).

Moreover, for the optimal parameter $\alpha^{*}$ and $\beta^{*}$, we have

$$
\begin{aligned}
\beta^{*}-\alpha^{*}-2 \lambda_{\min } & =2\left[\frac{\left(P_{\lambda}-P_{\mu}\right)-\lambda_{\min }\left(S_{\mu}+S_{\curlywedge}\right)}{\left(S_{\mu}+S_{\curlywedge}\right)}\right] \\
& =-\frac{2\left(\lambda_{\min }+\mu_{\min }\right)\left(\lambda_{\min }+\mu_{\max }\right)}{\left(S_{\mu}+S_{\curlywedge}\right)} \quad(\text { from }(3.14)) \\
& <0 .
\end{aligned}
$$

By similarity computation, we obtain $\beta^{*}-\alpha^{*}-2 \mu_{\min }>0$. So, the parameters $\alpha^{*}$ and $\beta^{*}$ satisfy the relation (3.20).

Finally, by denoting

$$
\Delta=\sqrt{\left(P_{\mu}-P_{\lambda}\right)^{2}+\left(S_{\lambda}+S_{\mu}\right)\left(S_{\mu} P_{\lambda}+S_{\lambda} P_{\mu}\right)},
$$


and substituting $\alpha^{*}$ and $\beta^{*}$ in (3.10) and using the relations (3.11)-(3.15), we obtain the optimal bound

$$
\begin{aligned}
\delta^{*}\left(\alpha^{*}, \beta^{*}\right) & =\frac{\alpha^{*}-\mu_{\min }}{\beta^{*}+\mu_{\min }} \times \frac{\beta^{*}-\lambda_{\min }}{\alpha^{*}+\lambda_{\min }} \\
& =\frac{\left(P_{\mu}-P_{\lambda}\right)+\sqrt{\Delta}-\mu_{\min }\left(S_{\mu}+S_{\curlywedge}\right)}{\left(P_{\lambda}-P_{\mu}\right)+\sqrt{\Delta}+\mu_{\min }\left(S_{\mu}+S_{\curlywedge}\right)} \times \frac{\left(P_{\lambda}-P_{\mu}\right)+\sqrt{\Delta}-\lambda_{\min }\left(S_{\mu}+S_{\curlywedge}\right)}{\left(P_{\mu}-P_{\curlywedge}\right)+\sqrt{\Delta}+\lambda_{\min }\left(S_{\mu}+S_{\curlywedge}\right)} \\
& =\frac{\sqrt{\Delta}-\left(\mu_{\min }+\lambda_{\min }\right)\left(\mu_{\min }+\lambda_{\max }\right)}{\sqrt{\Delta}+\left(\mu_{\min }+\lambda_{\min }\right)\left(\mu_{\min }+\lambda_{\max }\right)} \times \frac{\sqrt{\Delta}-\left(\lambda_{\min }+\mu_{\min }\right)\left(\lambda_{\min }+\mu_{\max }\right)}{\sqrt{\Delta}+\left(\lambda_{\min }+\mu_{\min }\right)\left(\lambda_{\min }+\mu_{\max }\right)} \\
& =\frac{\left(\mu_{\max }+\lambda_{\min }\right)\left(\lambda_{\max }+\mu_{\min }\right)-\sqrt{\Delta}}{\left(\mu_{\max }+\lambda_{\min }\right)\left(\lambda_{\max }+\mu_{\min }\right)+\sqrt{\Delta}} \\
& =\frac{\sqrt{\left(\lambda_{\max }+\mu_{\min }\right)\left(\mu_{\max }+\lambda_{\min }\right) /\left(\lambda_{\max }+\mu_{\max }\right)\left(\lambda_{\min }+\mu_{\min }\right)}-1}{\sqrt{\left(\lambda_{\max }+\mu_{\min }\right)\left(\mu_{\max }+\lambda_{\min }\right) /\left(\lambda_{\max }+\mu_{\max }\right)\left(\lambda_{\min }+\mu_{\min }\right)}+1} .
\end{aligned}
$$

Remark 3.5. We remark that if the eigenvalues of matrices $C$ and $D$ contain in $\Omega=\left[\gamma_{\min }, \gamma_{\max }\right]$, and we estimate $\delta(\alpha, \beta)$, as [6], by

$$
\bar{\delta}(\alpha, \beta)=\max _{\gamma \in \Omega}\left(\left|\frac{\beta-\gamma}{\alpha+\gamma}\right| \max _{\gamma \in \Omega}\left|\frac{\alpha-\gamma}{\beta+\gamma}\right|\right)
$$

then by taking $\beta=\alpha$, we obtain

$$
\begin{gathered}
\alpha^{*}=\sqrt{\gamma_{\min } \gamma_{\max }}, \\
\bar{\delta}\left(\alpha^{*}\right)=\frac{\gamma_{\min }+\gamma_{\max }-2 \sqrt{\gamma_{\min } \gamma_{\max }}}{\gamma_{\min }+\gamma_{\max }+2 \sqrt{\gamma_{\min } \gamma_{\max }}},
\end{gathered}
$$

which are the same as those given in [6] for Hermitian positive definite matrix $A$.

\section{ACSCS Iteration Method for the BTTB Matrices}

In this section we extend our method to block-Toeplitz-Toeplitz-block (BTTB) matrices of the form

$$
A=\left(\begin{array}{cccc}
A_{0} & A_{1} & \cdots & A_{m-1} \\
A_{1} & A_{0} & \cdots & A_{m-2} \\
\vdots & & \ddots & \vdots \\
A_{m-1} & A_{m-2} & \cdots & A_{0}
\end{array}\right)
$$


with

$$
A_{j}=\left(\begin{array}{cccc}
a_{j, 0} & a_{j, 1} & \cdots & a_{j, n-1} \\
a_{j, 1} & a_{j, 0} & \cdots & a_{j, n-2} \\
\vdots & & \ddots & \vdots \\
a_{j, n-1} & a_{j, n-2} & \cdots & a_{j, 0}
\end{array}\right) .
$$

Similar to the Toeplitz matrix, the BTTB matrix A possesses a splitting [11]:

$$
A=C_{c}+C_{s}+S_{c}+S_{s}
$$

where $C_{c}$ is a block-circulant-circulant-block (BCCB) matrix, $C_{s}$ is a block-circulant-skewcirculant-block (BCSB) matrix, $S_{c}$ is a block-skew-circulant-circulant-block (BSCB) matrix, and $S_{S}$ is a block-skew-circulant-skew-circulant block (BSSB) matrix. We note that the matrices $C_{c}, C_{s}, S_{c}$, and $S_{s}$ can be diagonalized by $F \otimes F, F \otimes \widehat{F}, \widehat{F} \otimes F$, and $\widehat{F} \otimes \widehat{F}$, respectively. Therefore, the systems of linear equations with coefficient matrices $\left(\alpha_{1,1} I+C_{c}\right),\left(\alpha_{1,2} I+\right.$ $\left.C_{s}\right),\left(\alpha_{2,1} I+S_{c}\right)$, and $\left(\alpha_{2,2} I+S_{s}\right)$, where $\alpha_{i, j}$ for $i, j=1,2$ are positive constants, can be solved efficiently using FFTs. The total number of operations required for each step of the method is $O(n m \log (n m))$ where $n m$ is the size of the BTTB matrix $A$. Based on the splitting of $A$ given in (4.3), the ACSCS iteration is as follows.

The ACSCS iteration method for BTTB matrix: given an initial guess $x^{(0)}$, for $k=$ $0,1,2, \ldots$, until $x^{(k)}$ converges, compute

$$
\begin{aligned}
\left(\alpha_{1,1} I+C_{c}\right) x^{(k+1 / 4)} & =\left(\alpha_{1,1} I-C_{s}-S_{c}-S_{s}\right) x^{(k)}+b, \\
\left(\alpha_{1,2} I+C_{s}\right) x^{(k+2 / 4)} & =\left(\alpha_{1,2} I-C_{c}-S_{c}-S_{s}\right) x^{(k+1 / 4)}+b, \\
\left(\alpha_{2,1} I+S_{c}\right) x^{(k+3 / 4)} & =\left(\alpha_{2,2} I-S_{s}-C_{c}-C_{s}\right) x^{(k+2 / 4)}+b, \\
\left(\alpha_{2,2} I+S_{s}\right) x^{(k+1)} & =\left(\alpha_{2,2} I-S_{c}-C_{c}-C_{s}\right) x^{(k+3 / 4)}+b,
\end{aligned}
$$

where $\alpha_{i, j}, i, j=1,2$ are given positive constants.

In the sequel, we need the following definition and results.

Definition 4.1 (see [12]). A splitting $A=M-N$ is called $P$-regular if $M^{H}+N$ is Hermitian positive definite.

Theorem 4.2 (see [13]). Let $A$ be Hermitian positive definite. Then $A=M-N$ is a P-regular splitting if and only if $\left\|M^{-1} N\right\|_{A}<1$.

Lemma 4.3 (see [14]). Suppose $A, B \in \mathbb{C}^{n \times n}$ be two Hermitian matrices, then

$$
\begin{gathered}
\lambda_{\max }(A+B) \leq \lambda_{\max }(A)+\lambda_{\max }(B), \\
\lambda_{\min }(A+B) \geq \lambda_{\min }(A)+\lambda_{\min }(B),
\end{gathered}
$$


where $\lambda_{\min }(X)$ and $\lambda_{\max }(X)$ denote the minimum and the maximum eigenvalues of matrix $X$, respectively.

Now we give the main results as follows.

Theorem 4.4. Let $A$ be a Hermitian positive definite BTTB matrix, and $C_{c}, C_{s}, S_{c}$, and $S_{s}$ be its $B C C B, B C S B, B S C B$, and $B S S B$ parts, and $\alpha_{i, j}, i, j=1,2$ be positive constants. Then the iteration matrix $M$ of the ACSCS method for BTTB matrices is

$$
\begin{aligned}
M= & \left(\alpha_{2,2} I+S_{s}\right)^{-1}\left(\alpha_{2,2} I-S_{c}-C_{c}-C_{s}\right)\left(\alpha_{2,1} I+S_{c}\right)^{-1}\left(\alpha_{2,1} I-S_{s}-C_{c}-C_{s}\right) \\
& \times\left(\alpha_{1,2} I+C_{s}\right)^{-1}\left(\alpha_{1,2} I-C_{c}-S_{c}-S_{s}\right)\left(\alpha_{1,1} I+C_{c}\right)^{-1}\left(\alpha_{1,1} I-C_{s}-S_{c}-S_{s}\right) .
\end{aligned}
$$

And if

$$
\begin{aligned}
& \alpha_{1,1}>\frac{\lambda_{\max }\left(C_{s}\right)+\lambda_{\max }\left(S_{c}\right)+\lambda_{\max }\left(S_{s}\right)-\lambda_{\min }\left(C_{c}\right)}{2}\left(\equiv \widetilde{\alpha}_{1,1}\right), \\
& \alpha_{1,2}>\frac{\lambda_{\max }\left(C_{c}\right)+\lambda_{\max }\left(S_{c}\right)+\lambda_{\max }\left(S_{s}\right)-\lambda_{\min }\left(C_{s}\right)}{2}\left(\equiv \widetilde{\alpha}_{1,2}\right), \\
& \alpha_{2,1}>\frac{\lambda_{\max }\left(S_{s}\right)+\lambda_{\max }\left(C_{c}\right)+\lambda_{\max }\left(C_{s}\right)-\lambda_{\min }\left(S_{c}\right)}{2}\left(\equiv \widetilde{\alpha}_{2,1}\right), \\
& \alpha_{2,2}>\frac{\lambda_{\max }\left(S_{c}\right)+\lambda_{\max }\left(C_{c}\right)+\lambda_{\max }\left(C_{s}\right)-\lambda_{\min }\left(S_{s}\right)}{2}\left(\equiv \widetilde{\alpha}_{2,2}\right),
\end{aligned}
$$

then the spectral radius $\rho(M)<1$, and the ACSCS iteration converges to the unique solution $x^{*} \in \mathbb{C}^{n}$ of the system of linear equations (1.1) for all initial vectors $x^{(0)} \in \mathbb{C}^{n}$.

Proof. By the definitions of BCCB, BCSB, BSCB, and BSSB parts of $A$, the matrices $C_{c}, C_{s}, S_{c}$, and $S_{s}$ are Hermitian. Let us consider the Hermitian matrices

$$
\begin{array}{ll}
M_{1}=\alpha_{1,1} I+C_{c}, & N_{1}=\alpha_{1,1} I-C_{s}-S_{c}-S_{s}, \\
M_{2}=\alpha_{1,2} I+C_{s}, & N_{2}=\alpha_{1,2} I-C_{c}-S_{c}-S_{s}, \\
M_{3}=\alpha_{2,1} I+S_{c}, & N_{3}=\alpha_{2,1} I-S_{s}-C_{c}-C_{s}, \\
M_{4}=\alpha_{2,2} I+S_{s}, & N_{4}=\alpha_{2,2} I-S_{c}-C_{c}-C_{s} .
\end{array}
$$

Since $A$ is Hermitian positive definite, it follows that

$$
M_{i}-N_{i}>0, \quad \text { for } i=1,2,3,4 .
$$

By the assumptions (4.7) and Lemma 4.3, we have also

$$
M_{i}+N_{i}>0, \quad \text { for } i=1,2,3,4 \text {. }
$$


The relations (4.9) and (4.10) imply that $M_{i}>0$, for $i=1,2,3$, 4. So, the matrices $M_{i}>0$, for $i=1,2,3,4$, are nonsingular and we get (4.6). In addition, the splittings $A=M_{i}-N_{i}$, $i=$ $1,2,3,4$ are $P$-regular and by Theorem 4.2 , we have

$$
\left\|M_{i}^{-1} N_{i}\right\|_{A}<1, \quad \text { for } i=1,2,3,4
$$

Finally, by using (4.11), we can obtain

$$
\|M\|_{A} \leq\left\|M_{1}^{-1} N_{1}\right\|_{A}\left\|M_{2}^{-1} N_{2}\right\|_{A}\left\|M_{3}^{-1} N_{3}\right\|_{A}\left\|M_{4}^{-1} N_{4}\right\|_{A}<1
$$

which completes the proof.

\section{Numerical Experiments}

In this section, we compare the ACSCS method with CSCS and CG methods for 1D and 2D Toeplitz problems. We used the vector of all ones for the right-hand side vector $b$. All tests are started from the zero vector, performed in MATLAB 7.6 with double precision, and terminated when

$$
\frac{\left\|r^{(k)}\right\|_{2}}{\left\|r^{(0)}\right\|_{2}} \leq 10^{-7},
$$

or when the number of iterations is over 1000. This case is denoted by the symbol "_". Here $r^{(k)}$ is the residual vector of the system of linear equation (1.1) at the current iterate $x^{(k)}$, and $r^{(0)}$ is the initial one.

For 1D Toeplitz problems (Examples 5.1-5.3), our comparisons are done for the number of iterations of the CG, CSCS, and ACSCS methods (denoted by "IT") and the elapsed CPU time (denoted by "CPU"). All numerical results are performed for $n=$ $16,32,64,128,256,512,1024$. The corresponding numerical results are listed in Tables 1-4. In these tables $\lambda_{\min }$ and $\mu_{\min }$ represent the minimum eigenvalue of matrices $C$ and $S$, respectively. Note that the CPU time in these tables does not account those for computing the iteration parameters. For ACSCS method, $\alpha^{*}$ and $\beta^{*}$ are computed by (3.16) and (3.17), respectively. And for CSCS method, $\alpha^{*}$ is computed by (3.34).

Example 5.1 (see [10]). In this example $A_{n}$ is symmetric positive definite Toeplitz matrix,

$$
a_{k}= \begin{cases}\frac{4 \pi^{2}(-1)^{k}}{k^{2}}-\frac{24(-1)^{k}}{k^{4}}, & k \neq 0, \\ 1+\frac{\pi^{5}}{5}, & k=0\end{cases}
$$

with generating function $f(\theta)=\theta^{4}+1, \theta \in[-\pi, \pi]$. Numerical results for this example are listed in Table 1. 
Table 1: Numerical results of Example 5.1.

\begin{tabular}{lcccccccc}
\hline$n$ & $\lambda_{\text {min }}$ & \multicolumn{1}{l}{$\mu_{\text {min }}$} & \multicolumn{3}{c}{ IT } & & \multicolumn{3}{c}{ CPU } \\
\hline 16 & 0.4183 & 0.5825 & 8 & 35 & 37 & 0.0077 & 0.0101 & 0.0095 \\
32 & 0.4801 & 0.5199 & 20 & 39 & 39 & 0.0085 & 0.0106 & 0.0100 \\
64 & 0.4951 & 0.5049 & 37 & 40 & 39 & 0.0098 & 0.0108 & 0.0106 \\
128 & 0.4988 & 0.5012 & 55 & 40 & 40 & 0.0108 & 0.0115 & 0.0128 \\
256 & 0.4997 & 0.5003 & 67 & 40 & 40 & 0.0138 & 0.0142 & 0.0144 \\
512 & 0.4991 & 0.5001 & 70 & 40 & 40 & 0.0189 & 0.0203 & 0.0205 \\
1024 & 0.5000 & 0.5000 & 71 & 40 & 40 & 0.0283 & 0.0316 & 0.0292 \\
\hline
\end{tabular}

Table 2: Numerical results of Example 5.2.

\begin{tabular}{lcccccccc}
\hline$n$ & $\lambda_{\text {min }}$ & \multicolumn{1}{l}{$\mu_{\text {min }}$} & CG & CSCS & ACSCS & CG & CSCS & ACSCS \\
\hline 16 & 0.4478 & 0.4177 & 12 & 8 & 8 & 0.0074 & 0.0079 & 0.0079 \\
32 & 0.4419 & 0.4247 & 15 & 9 & 9 & 0.0076 & 0.0087 & 0.0084 \\
64 & 0.4377 & 0.4292 & 17 & 10 & 10 & 0.0079 & 0.0090 & 0.0091 \\
128 & 0.4355 & 0.4315 & 19 & 11 & 11 & 0.0083 & 0.0094 & 0.0093 \\
256 & 0.4344 & 0.4325 & 20 & 12 & 12 & 0.0095 & 0.0106 & 0.0103 \\
512 & 0.4339 & 0.4330 & 21 & 13 & 13 & 0.0101 & 0.0111 & 0.0111 \\
1024 & 0.4337 & 0.4333 & 22 & 14 & 14 & 0.0148 & 0.0161 & 0.0150 \\
\hline
\end{tabular}

Table 3: Numerical results of Example 5.3 for $\beta=10, \gamma=0.5$.

\begin{tabular}{|c|c|c|c|c|c|c|c|c|}
\hline \multirow{2}{*}{$n$} & \multirow{2}{*}{$\lambda_{\min }$} & \multirow{2}{*}{$\mu_{\min }$} & \multicolumn{3}{|c|}{ IT } & \multicolumn{3}{|c|}{ CPU } \\
\hline & & & CG & CSCS & ACSCS & CG & CSCS & ACSCS \\
\hline 16 & 1.0621 & 0.1280 & 8 & 20 & 10 & 0.0072 & 0.0095 & 0.0080 \\
\hline 32 & 0.7746 & -0.0251 & 16 & - & 13 & 0.0077 & - & 0.0085 \\
\hline 64 & 0.6285 & -0.1005 & 23 & - & 15 & 0.0082 & - & 0.0090 \\
\hline 128 & 0.5169 & -0.1379 & 28 & - & 18 & 0.0096 & - & 0.0096 \\
\hline 256 & 0.4410 & 0.1485 & 32 & 20 & 15 & 0.0107 & 0.0117 & 0.0100 \\
\hline 512 & 0.3841 & 0.1207 & 34 & 23 & 16 & 0.0128 & 0.0139 & 0.0125 \\
\hline 1024 & 0.3444 & 0.1067 & 35 & 24 & 17 & 0.0173 & 0.0245 & 0.0185 \\
\hline
\end{tabular}

Table 4: Numerical results of Example 5.3 for $\beta=10, \gamma=0.1$.

\begin{tabular}{lcccccccc}
\hline$n$ & $\lambda_{\text {min }}$ & $\mu_{\min }$ & \multicolumn{3}{c}{ IT } & & \multicolumn{3}{c}{ CPU } \\
& & & CG & CSCS & ACSCS & CG & CSCS & ACSCS \\
\hline 16 & 0.8963 & -0.0771 & 8 & - & 12 & 0.0077 & - & 0.0082 \\
32 & 0.5967 & -0.2367 & 16 & - & 18 & 0.0082 & - & 0.0090 \\
64 & 0.4444 & 0.1194 & 26 & 22 & 16 & 0.0090 & 0.0092 & 0.0093 \\
128 & 0.3282 & 0.0025 & 36 & - & 20 & 0.0102 & - & 0.0105 \\
256 & 0.2490 & 0.0473 & 47 & - & 27 & 0.0123 & - & 0.0130 \\
512 & 0.1898 & -0.0012 & 59 & - & 29 & 0.0170 & - & 0.0171 \\
1024 & 0.1484 & 0.0125 & 68 & - & 31 & 0.0279 & - & 0.0297 \\
\hline
\end{tabular}


Example 5.2 (see [15]). Let $A_{n}$ be a Hermitian positive definite Toeplitz matrix,

$$
a_{k}= \begin{cases}\frac{1+\sqrt{-1}}{(1+k)^{1.1}}, & k>0, \\ 2, & k=0, \\ \bar{a}_{-} k, & k<0 .\end{cases}
$$

The associated generating function is $f(\theta)=2 \sum_{k=0}^{\infty}(\sin (k \theta)+\cos (k \theta)) /(1+k)^{1.1}, \theta \in[0,2 \pi]$. Numerical results of this example are presented in Table 2.

Example 5.3 (see [10]). In this example $A_{n}$ is Hermitian positive definite Toeplitz matrix,

$$
a_{k}= \begin{cases}\frac{-\sqrt{-1}(\beta-\gamma)\left(1+(-1)^{k}\right),}{2 \pi k} & k>0, \\ (\beta+\gamma), & k=0, \\ \bar{a}_{-}, & k<0\end{cases}
$$

with generating function

$$
f_{\{\beta, \gamma\}}(\theta)= \begin{cases}\frac{\beta-\gamma}{\pi} \theta+\beta, & -\pi \leq \theta<0, \\ \frac{\beta-\gamma}{\pi} \theta+\gamma, & 0<\theta \leq \pi,\end{cases}
$$

where $\beta$ and $\gamma$ are the maximum and minimum values of $f_{\{\beta, \gamma\}}$ on $[-\pi, \pi]$, respectively. In Tables 3 and 4, numerical results are reported for $\beta=10, \gamma=0.5$ and $\beta=10, \gamma=0.1$, respectively.

In the following, we summarize the observation from Tables 1-4. In all cases, in terms of CPU time needed for convergence, the ACSCS converges at the same rate that the CG method converges. However, the number of ACSCS iterations is less than that of CG iterations required for convergence. The convergence behavior of ACSCS method, in terms of the number of iterations and CPU time needed for convergence, is similar to that of CSCS method when $\lambda_{\min }$ and $\mu_{\min }$ are positive and not too small (all the cases in Tables 1 and 2). Moreover, we observe that, when $\lambda_{\min }$ and $\mu_{\min }$ are too small or negative (the cases $n=32,64,128$ in Table 3 and the cases $n \neq 64$ in Table 4), the ACSCS method converges at the same rate that the CG converges, but the CSCS method does not converge. These results imply that the computational efficiency of the ACSCS iteration method is similar to that of the CG method and is higher than that of the CSCS iterations.

For 2D Toeplitz problems, we tested three problems of the form given in (4.1) with the diagonals of the blocks $A_{j}$. The diagonals of $A_{j}$ are given by the generating sequences 
Table 5: Numerical results of 2D examples.

\begin{tabular}{|c|c|c|c|c|c|c|c|c|c|}
\hline \multirow{2}{*}{$n$} & \multicolumn{3}{|c|}{ Sequence (a) } & \multicolumn{3}{|c|}{ Sequence (b) } & \multicolumn{3}{|c|}{ Sequence (c) } \\
\hline & CG & ACSCS & CSCS & $\mathrm{CG}$ & ACSCS & CSCS & CG & ACSCS & CSCS \\
\hline 8 & 15 & 35 & 42 & 15 & 30 & 36 & 10 & 17 & 19 \\
\hline 16 & 28 & 50 & 57 & 27 & 43 & 49 & 16 & 25 & 28 \\
\hline 32 & 37 & 60 & 65 & 35 & 51 & 56 & 23 & 34 & 37 \\
\hline 64 & 45 & 64 & 68 & 41 & 54 & 58 & 30 & 41 & 44 \\
\hline 128 & 49 & 63 & 66 & 46 & 54 & 56 & 37 & 46 & 49 \\
\hline
\end{tabular}

Table 6: Numerical results of 2D examples for ACSCS method with $\alpha_{1,1}=0.5\left(<\tilde{\alpha}_{1,1}\right)$.

\begin{tabular}{lccc}
\hline$n$ & Sequence (a) & Sequence (b) & \multicolumn{2}{c}{ Sequence (c) } \\
& IT & IT & IT \\
\hline 8 & 15 & 14 & 13 \\
16 & 15 & 15 & 14 \\
32 & 17 & 17 & 15 \\
64 & 20 & 20 & 16 \\
128 & 22 & 22 & 17 \\
\hline
\end{tabular}

Table 7: Numerical results of 2D examples for CSCS method with optimal $\alpha$.

\begin{tabular}{lcccccc}
\hline$n$ & \multicolumn{2}{c}{ Sequence (a) } & \multicolumn{2}{c}{ Sequence (b) } & \multicolumn{2}{c}{ Sequence (c) } \\
& $\alpha^{*}$ & IT & $\alpha^{*}$ & IT & $\alpha^{*}$ & IT \\
\hline 8 & 2.48 & 26 & 2.31 & 23 & 1.18 & 15 \\
16 & 3.75 & 35 & 3.53 & 31 & 1.79 & 20 \\
32 & 5.14 & 42 & 4.65 & 36 & 2.41 & 25 \\
64 & 6.33 & 44 & 5.70 & 39 & 3.17 & 30 \\
128 & 7.39 & 44 & 6.74 & 39 & 3.93 & 34 \\
\hline
\end{tabular}

(see [10])
(a) $a_{j, i}=1 /(j+1)(|i|+1)^{1+.1(j+1)}, j \geq 0, i=0, \pm 1, \pm 2, \ldots$,
(b) $a_{j, i}=1 /(j+1)^{1.1}(|i|+1)^{1+.1(j+1)}, j \geq 0, i=0, \pm 1, \pm 2, \ldots$,
(c) $a_{j, i}=1 /(j+1)^{2.1}+(|i|+1)^{2.1}, j \geq 0, i=0, \pm 1, \pm 2, \ldots$

The generating sequences (b) and (c) are absolutely summable while (a) is not. Our comparisons are done for the number of iterations of the CG, CSCS, and ACSCS methods (denoted by "IT"). All numerical results are performed for $n=16,32,64,128$. The corresponding numerical results are listed in Table 5. For ACSCS method, parameters $\alpha_{i, j}=\tilde{\alpha}_{i, j}$, $i, j=1,2$ are used. For CSCS method, we used $\alpha=\max _{i, j=1}^{2}\left\{\alpha_{i, j}\right\}$. Table 5 shows that, in all cases, the number of ACSCS iterations required for convergence is less than that of CSCS method and more than that of CG method. We mention that the relations (4.7) are sufficient conditions for convergence of ACSCS iteration for BTTB matrices. The numerical experiments show that the convergence behavior of ACSCS method, in terms of the number of iterations needed for convergence, is better than that of CG and CSCS methods if one of the parameters $\alpha_{i, j}, i, j=1,2$ is chosen less than the corresponding lower bound $\tilde{\alpha}_{i, j}$ given in Theorem 4.2. 
Table 6 presents the results which are obtained for the ACSCS method with $\alpha_{1,1}=0.5\left(<\widetilde{\alpha}_{1,1}\right)$, $\alpha_{1,2}=\tilde{\alpha}_{1,2}, \alpha_{2,1}=\tilde{\alpha}_{2,1}$, and $\alpha_{2,2}=\tilde{\alpha}_{2,2}$. Table 7 presents the results obtained for CSCS method with the optimal parameter $\alpha$, obtained computationally by trial and error. As we observe, from Tables 5-7, the number of ACSCS iterations required for convergence is less than that of CG and CSCS methods.

These results imply that the computational efficiency of the ACSCS iteration method is similar to that of the CG method and is higher than that of the CSCS iterations.

\section{Conclusion}

In this paper, a new iteration method for the numerical solution of Hermitian positive definite Toeplitz systems of linear equations has been described. This method, which called ACSCS method, is a two- (four-) parameter generation of the CSCS of Ng for 1D (2D) problems and is based on the circulant and the skew circulant splitting of the Toeplitz matrix. We theoretically studied the convergence properties of the ACSCS method. Moreover, the contraction factor of the ACSCS iteration and its optimal parameters are derived. Theoretical considerations and numerical examples indicate that the splitting method is extremely effective when the generation function is positive. Numerical results also showed that the computational efficiency of the ACSCS iteration method is similar to that of the CG method and is higher than that of the CSCS iteration method.

\section{Acknowledgment}

The authors would like to thank the referee and the editor very much for their many valuable and thoughtful suggestions for improving this paper.

\section{References}

[1] P. Delsarte and Y. V. Genin, "The split Levinson algorithm," IEEE Transactions on Acoustics, Speech, and Signal Processing, vol. 34, no. 3, pp. 470-478, 1986.

[2] N. Levinson, "The Wiener RMS (root mean square) error criterion in filter design and prediction," Journal of Mathematics and Physics, vol. 25, pp. 261-278, 1947.

[3] W. F. Trench, "An algorithm for the inversion of finite Toeplitz matrices," vol. 12, pp. 515-522, 1964.

[4] G. S. Ammar and W. B. Gragg, "Superfast solution of real positive definite Toeplitz systems," SIAM Journal on Matrix Analysis and Applications, vol. 9, no. 1, pp. 61-76, 1988.

[5] G. Strang, "Proposal for Toeplitz matrix calculations," Studies in Applied Mathematics, vol. 74, no. 2, pp. 171-176, 1986.

[6] M. K. Ng, "Circulant and skew circulant splitting methods for Toeplitz systems," Journal of Computational and Applied Mathematics, vol. 159, no. 1, pp. 101-108, 2003.

[7] C. Gu and Z. Tian, "On the HSS iteration methods for positive definite Toeplitz linear systems," Journal of Computational and Applied Mathematics, vol. 224, no. 2, pp. 709-718, 2009.

[8] Z. Z. Bai, G. H. Golub, and M. K. Ng, "Hermitian and skew-Hermitian splitting methods for nonHermitian positive definite linear systems," SIAM Journal on Matrix Analysis and Applications, vol. 24, no. 3, pp. 603-626, 2003.

[9] T. K. Ku and C. C. J. Kuo, "Design and analysis of Toeplitz preconditioners," IEEE Transactions on Signal Processing, vol. 40, no. 1, pp. 129-141, 1992.

[10] M. K. Ng, Iterative Methods for Toeplitz Systems, Oxford University Press, New York, NY, USA, 2004.

[11] R. H. Chan and M. K. Ng, "Conjugate gradient methods for Toeplitz systems," SIAM Review, vol. 38, no. 3, pp. 427-482, 1996. 
[12] J. M. Ortega, Numerical Analysis. A Second Course, Academic Press, New York, NY, USA, 1972.

[13] A. Frommer and D. B. Syzld, "Weighted max norms, splittings, and overlapping additive Schwarz iterations," Numerische Mathematik, vol. 5, p. 4862, 1997.

[14] R. A. Horn and C. R. Johnson, Matrix Analysis, Cambridge University Press, New York, NY, USA, 1985.

[15] R. H. Chan, "Circulant preconditioners for Hermitian Toeplitz systems," SIAM Journal on Matrix Analysis and Applications, vol. 10, no. 4, pp. 542-550, 1989. 


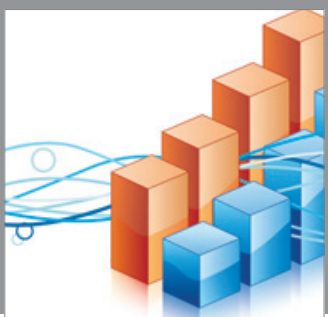

Advances in

Operations Research

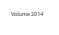

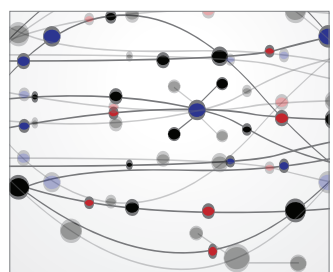

\section{The Scientific} World Journal
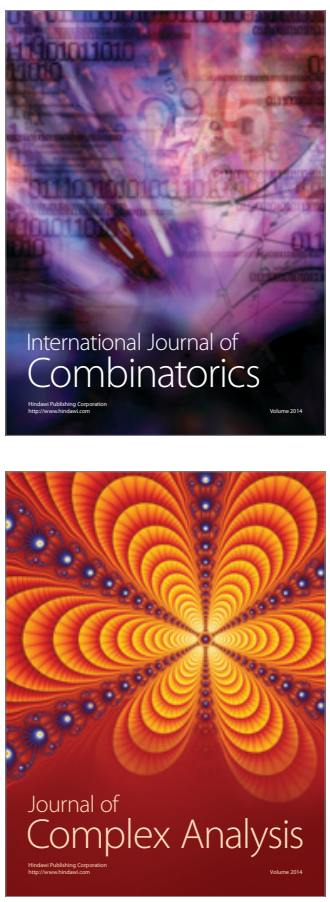

International Journal of

Mathematics and

Mathematical

Sciences
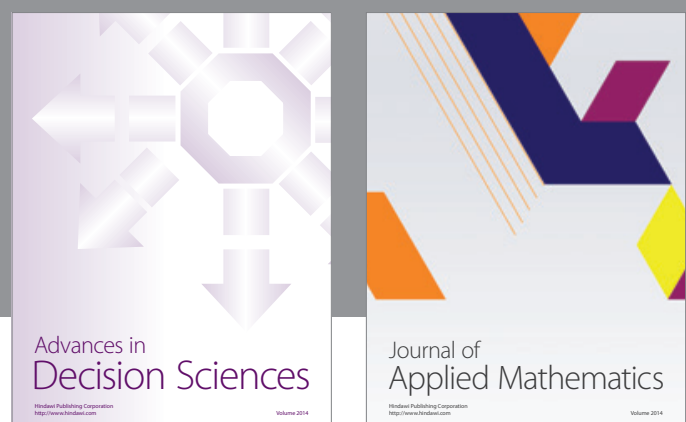

Journal of

Applied Mathematics
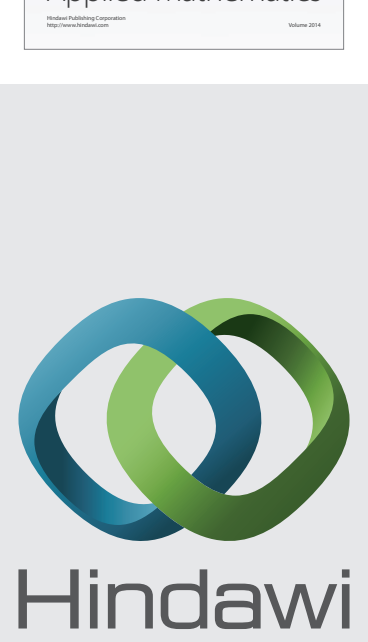

Submit your manuscripts at http://www.hindawi.com
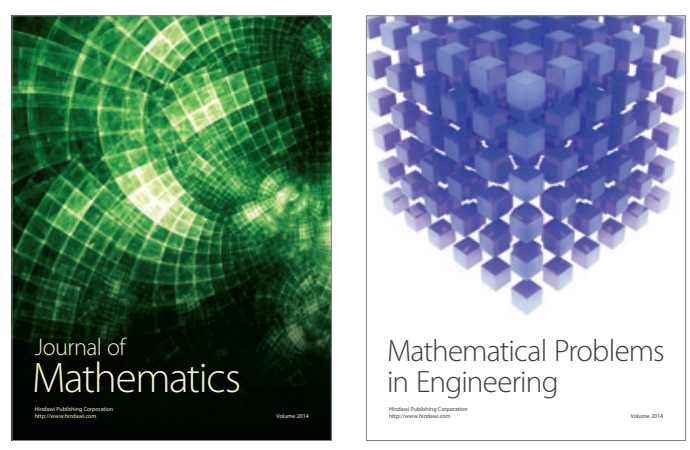

Mathematical Problems in Engineering
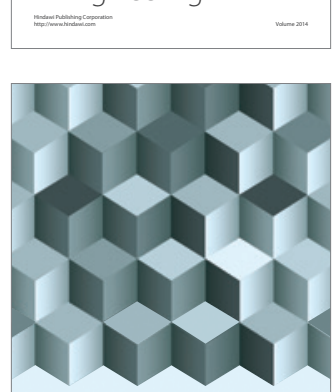

Journal of

Function Spaces
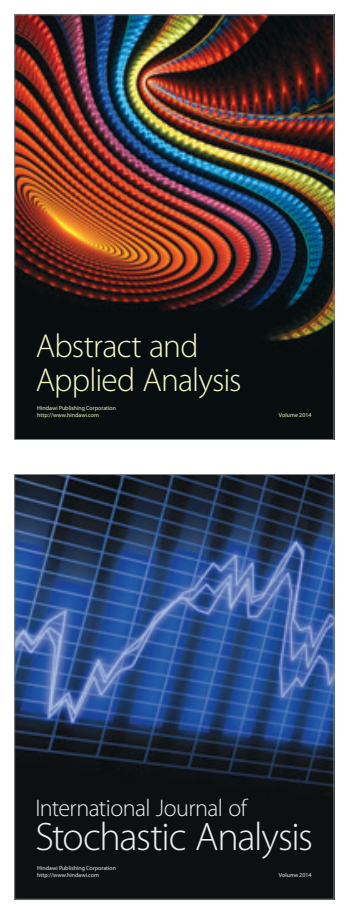

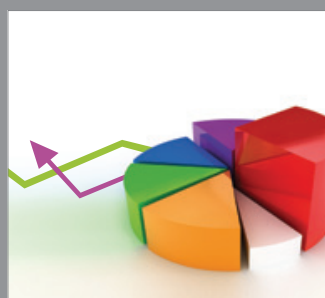

ournal of

Probability and Statistics

Promensencen
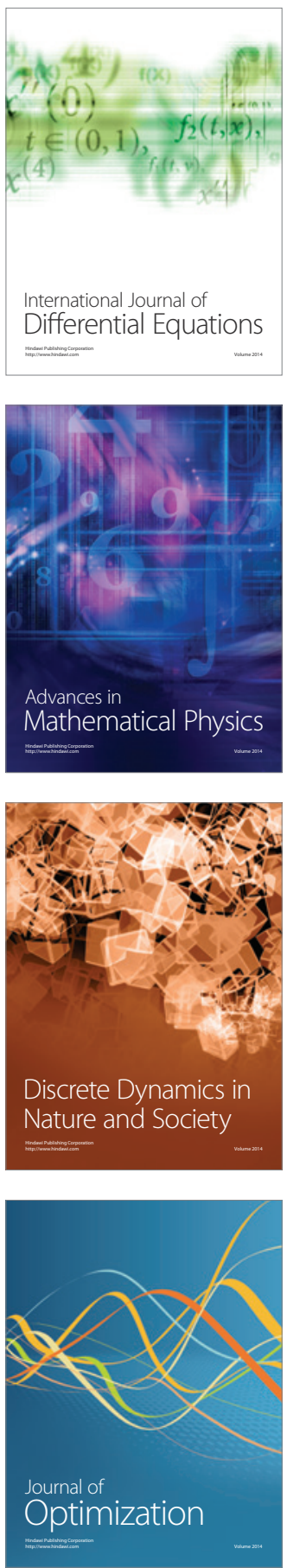